\title{
Embarazo ectópico cervical: Estudio de un caso clínico
}

\section{Cervical Ectopic Pregnancy: A Clinical Case Study}

Aída Isabel Jordán Bolaños. ${ }^{1}$, Cristina Anahí Mantilla P. ${ }^{2}$ \& Cristina Elizabeth Rodríguez. ${ }^{3}$

Recibido: 09-04-2020 / Revisado: 18-05-2020 / Aceptado: 13-06-2020 / Publicado: 03-07-2020

DOI: https://doi.org/10.33262/anatomiadigital.v3i3.1391

\begin{abstract}
.
Ectopic pregnancy is one of the health emergencies that patients require the greatest promptness in care, hence the interest in studying its traits and behaviors at the Ambato General Teaching Hospital in the province of Tungurahua. In this sense, the authors present the following research objective: Describe the features that characterize the procedure in the treatment of ectopic pregnancy. In order to fulfill the objective, the study of a previous clinical case of a 38-year-old female patient from the province was presented with no previous history of pregnancies and no evidence of hereditary factors of trauma in pregnancy. Different theoretical methods were used, such as the synthetic and inductive deductive analysis, which allowed us to analyze the
\end{abstract}

\section{Resumen.}

El embarazo ectópico es una de las emergencias sanitarias que requieren de una rápida atención, de ahí el interés por estudiar sus rasgos y comportamientos en el Hospital General Docente Ambato, de la provincia de Tungurahua. En este sentido los autores presentan como objetivo de investigación: Describir los rasgos que caracterizan el proceder en el tratamiento del embarazo ectópico cervical. Para darle cumplimiento al objetivo, se presentó el estudio de un caso clínico precedente de una paciente de 38 años propia de la provincia y sin antecedente de embarazos previos, factores hereditarios o de traumas en el embarazo. Se utilizaron diferentes métodos teóricos tales como, el analítico sintético e inductivo deductivo que permitieron analizar los rasgos $y$

\footnotetext{
${ }^{1}$ Médico de posgrado de la Especialización Medicina Familiar y Comunitaria II Cohorte Universidad Técnica de Ambato, Ambato, Ecuador, draisabeljordan@hotmail.com, (iD https://orcid.org/0000-0002-5131-8495

${ }^{2}$ Médico de posgrado de la Especialización Medicina Familiar y Comunitaria II Cohorte Universidad Técnica de Ambato, Ambato, Ecuador, maan.cada36@gmail.com, iD https://orcid.org/0000-0001-7443-3683

${ }^{3}$ Médico de posgrado de la Especialización Medicina Familiar y Comunitaria II Cohorte Universidad Técnica de Ambato, Ambato, Ecuador, dracristinarodriguezchicaiza@gmail.com, iD https://orcid.org/0000-0002-2759-3104
} 
Vol. 3, N³, p. 82-89, julio-septiembre, 2020

features and distinctive characteristics of this type of pregnancies, in addition to the structural systemic method it was possible to review the different types of treatments for these pregnancies. After performing an ultrasound and gynecological techniques, the main results were the application of a cesarean section that ended in hysterectomy.

Keywords: Ectopic pregnancy, hysterectomy, caesarean section, risk in pregnancy. características distintivas de este tipo de embarazo. Además, a través del método sistémico estructural se pudo revisar los diferentes tipos de tratamientos utilizados para esta patología. Luego de la realización de un ultrasonido y técnicas ginecológicas se obtuvo como principales resultados la aplicación de una cesárea que termina en histerectomía.

Palabras claves: Embarazo Ectópico, histerectomía, cesárea, riesgo en embarazo.

\section{Introducción.}

Cuando nos referimos al embarazo ectópico (Ecto asociado a la condición de fuera y topos asociado a lugar), no podemos dejar de mencionar que el embarazo tubárico es una de las afecciones más frecuentes del mundo. Y, está considerada dentro de las principales causas de mortalidad en mujeres en periodo de gestación. Por lo general su periodicidad se encuentra 1 entre 1800 a 2500 embarazos; representa 0,07 a $0,1 \%$ de todos los embarazos ectópicos (Varona; \& Martha Mohamed Abdelaziz, 2007). En el Hospital General Docente Ambato, estas cifras han sido rigurosamente controladas por el equipo ginecológico provincial, encontrando desde el año 2015 hasta marzo del 2020 un $0,03 \%$ de embarazo ectópico por cada 1000 casos de pacientes embarazadas.

Esto indica que, para el caso de Ambato, los ectópicos cervicales son los menos frecuentes y generalmente se presenta con una edad gestacional de 9,4 semanas, con poca evidencia. La causa de surgimiento de estos tipos de embarazo es desconocida (Varona; \& Martha Mohamed Abdelaziz, 2007). Para la investigación, esto indica que puede ser producido por la propia implantación y como es lógico el desarrollo del blastocito fuera del endometrial y factores relacionados como refieren diversos autores. Este criterio coincide con los expresado por Guerrero-Martínez; \& RivasLópez (2014) donde plantean que este tipo de embarazos está asociado a la implantación del blastocisto en cualquier lugar fuera de la cavidad endometrial. Las causas pueden ser multifactoriales, pero la principal se asocia con lesiones localizadas en la luz de la salpinx; lo que trae consigo la destrucción del epitelio y la formación de micro adherencias que en muchas ocasiones causan gran infección. A su vez, para Carrillo (2003) este tipo de embarazos es una de las causas de dolor abdominal de gran complicación. Por lo que se tiene que tener especial cuidado ya que, en varias ocasiones se confunde este fenómeno como sinónimo de embarazo extrauterino. Se debe entender que para el caso al cual nos estamos refiriendo, es más abarcador, 
pues hay gestaciones dentro del útero que son ectópica (intersticial y cervical). Se trata de una de las hemorragias de la primera mitad del embarazo cuya expresión clínica habitual es la tríada clásica de dolor abdominal, sangrado y amenorrea.(Carrillo, 2003). Studolsford citado por (Lozano \& García, 2003) propuso como suceso, que el óvulo fertilizado se pudiera trasladar más rápido y por este medio favoreciera su entrada al conducto cervical antes de anidar. Otros autores consideran es muy parecido a un embarazo normal y que como tal secreta hormona gonadotropina con un mantenimiento del cuerpo lúteo de la gestación y que a su vez mantiene la producción de estrógenos y progesterona en cantidades suficientes para ocasionar cambios fisiológicos en el organismo materno durante la primera fase del embarazo. (Arenas, 2011)

Los síntomas más recurrentes que se reportan en Ambato están relacionados con retrasos del el ciclo menstrual, síntomas relacionados con el embarazo, presencia de numerosos dolores abdominales, manchas de diferentes magnitudes y viscosidad en el sangrado de color oscuro. Esto tiene estrecha coincidencia con diferentes criterios médicos donde se presentan signos de dolor abdominal a la palpación (Varona; \& Martha Mohamed Abdelaziz, 2007), reacción peritoneal (Pérez, 2002), tacto a nivel vaginal, lugar donde se presenta una masa en condiciones tumorales (Pinto et al., 2015), fuerte dolor a la lateralización (Radan et al., 2020). Se debe recordar que no siempre la paciente muestra los mismos síntomas, por lo tanto hay que diferenciar los síntomas para descartar este padecimiento a tiempo, y, siempre con la ayuda de protocolos ya establecidos. Ejemplo de ello, se describe el uso de la laparotomía durante mucho tiempo como medida de tratamiento.

Esta técnica con el cursar de los años ha jugado un papel predominante en el tratamiento de este tipo de embarazo y aporta en la observación directa para la localización y constatación del tamaño del feto, las adherencias que presenta, el hemoperitoneo y la profundidad de la pelvis. Existe la posibilidad de seleccionar la vía para la cirugía o la técnica específica, y aún hasta el cirujano. Así, podemos realizarlo por laparoscopía, por laparotomía o con una mini-laparotomía con resultados satisfactorios. También, podremos sugerir en qué caso, por las condiciones pélvicas locales, se necesita la presencia en el acto quirúrgico de un profesor, o en cuál, por su sencillez, podemos dejar que sea operado por el residente, aunque siempre con la ayuda y asesoría de un especialista (Hidalgo, 1995).

\section{Presentación del caso clínico}

Paciente femenina de años, sin antecedentes obstétricos personales ni familiares, ingresó por emergencia del Hospital General Docente Ambato donde refiere haber tenido por más de hace 12 horas sangrado en abundante cantidad sin coágulos, acompañado de dolor tipo cólico de igual evolución. Luego de tomados todos los signos vitales y encontrarse la tensión arterial en 100/60, la saturación de oxígeno en 95 \% y las pulsaciones en 80 pul/min, se procede a realizar una prueba de $\beta$-hCG en sangre la cual fue positiva. Posteriormente es remitida a los servicios de ginecología, donde se le realiza un examen vaginal utilizando un espéculo, encontrándose un cuello de 
multípara con OCE cerrado (orificio cervical externo) y tumoraciones abundantes en el fondo del saco interior. Mediante el tacto vaginal se palpa partes blandas de aproximadamente $12 \mathrm{~cm}$ a nivel del cuello, acompañado de sangrado con presunta sospecha de un embarazo ectópico. Se realiza ultrasonido el cual reporta embarazo ectópico de implantación endocervical. (Fig 1)

Figura 1. Ultrasonido con reporte de embarazo ectópico de implantación endocervical

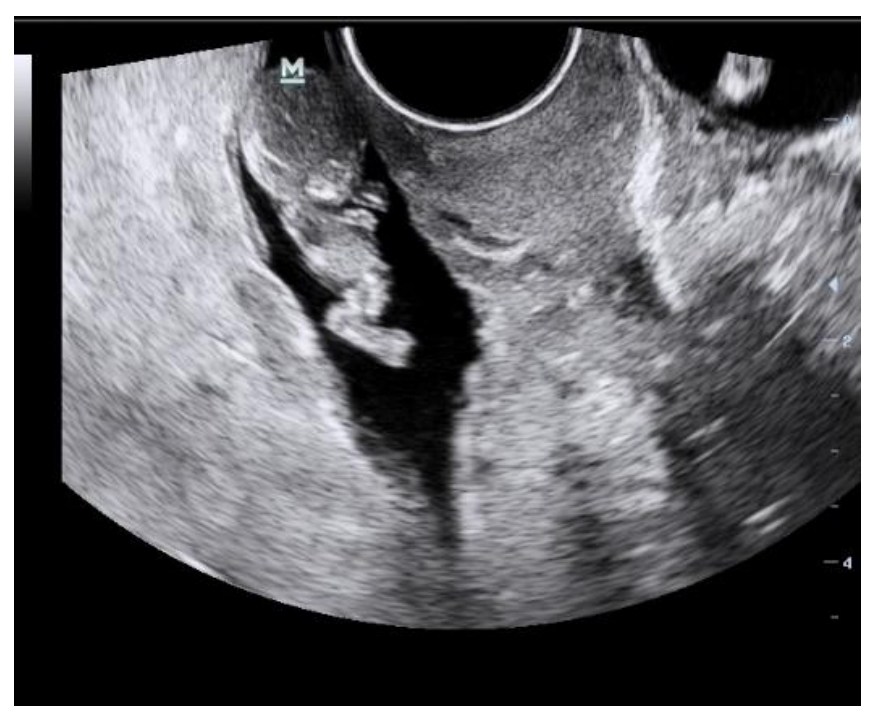

Fuente: Elaboración propia.

\section{Resultados.}

Al confirmar el diagnóstico se completa exámenes y se resuelve por cesárea, la misma que termina en histerectomía (Fig 2 y 3 )

Se trasladó a la paciente al quirófano donde se le realizó una dilatación del conducto cervical con tallos de Hegar sin ningún tipo de complicaciones. Luego se extrajeron paulatinamente restos con pinzas de Winter y se procedió a la legración de la cavidad con una legra mediana, con lo que paciente presenta sangrado en abundante cantidad que no se puede controlar por lo que se decide abrir.

Se realiza incisión Pfannenstiel, se profundiza hasta apertura de cavidad abdominal, posteriormente se procede a identificación, pinzamiento y exteriorización de útero. Luego se realiza identificación, pinzamiento, sección y ligadura de pedículo superior, apertura de hoja anterior y posterior de ligamento ancho y divulsión de repliegue vesicouterino y recto uterino. Identificación, pinzamiento, sección y ligadura de arterias uterinas bilateral. Identificación, pinzamiento, sección y ligadura de ligamentos cardinales bilateral, apertura de vagina y sección después de identificación de embarazo ectópico cervical. Finalmente se realiza la extracción de útero junto con cérvix, cierre de cúpula vaginal y peritonización de la misma. 
Figura 2. Cesárea e histerectomía 1

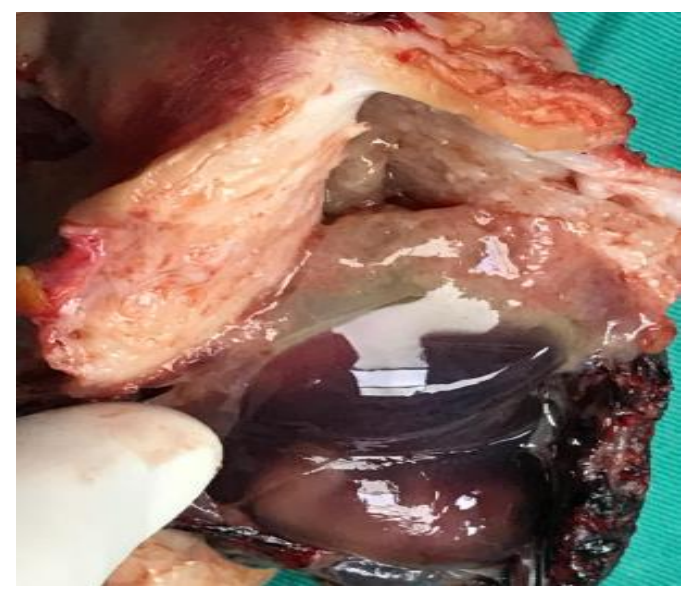

Fuente: Elaboración propia.

Figura 2. Cesárea e histerectomía 2

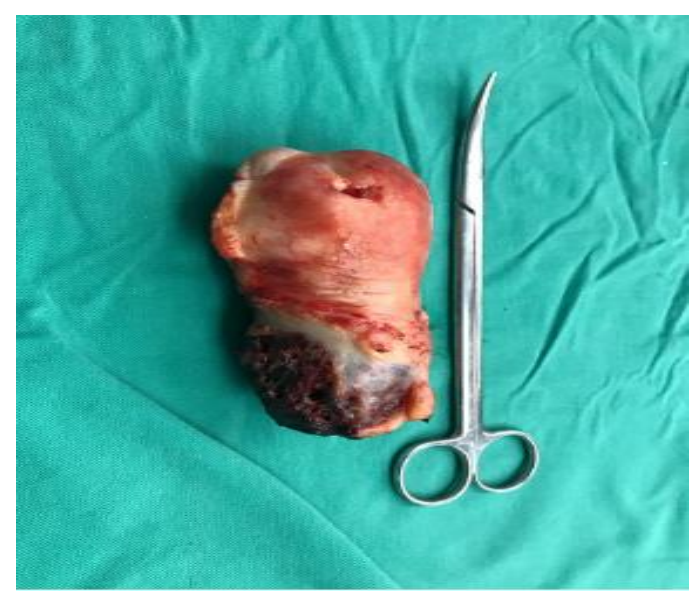

Fuente: Elaboración propia.

Discusión: el embarazo ectópico cervical puede causar la muerte si no se resuelve pronto(Cearra et al., 2009). Teniendo como factores de riesgo el antecedente de cesárea, enfermedad pélvica inflamatoria, técnicas de reproducción asistida. El eco transvaginal es el método más sensible para el diagnóstico precoz de la gestación ectópica con una sensibilidad del 87-99\% y una especificidad del 94-99,9\%. Los síntomas más frecuentes pueden ser molestias abdominales inespecíficas o escaso manchado vaginal, con amenorrea(Burgos J et al., 2004). El spotting vaginal es el signo más común, ya que solo un tercio de las pacientes presentan hemorragia vaginal abundante. Algunos autores consideran que una de las principales amenazas de este tipo de embarazo suele aumentar con la edad, siendo generalmente entre los 30 y los 40 años los signos más propensos (Cristina González et al., 2012). Para el caso que se presenta en la investigación de la paciente de 34 años el grupo de investigadores concuerda en los criterios antes planteados. 


\section{Conclusión.}

- El embarazo ectópico cervical, es un caso raro y grave de implantación del cigoto en el cérvix. Por tal motivo se debe conocer de su existencia y pensar en él, para hacer el diagnóstico temprano y poder instalar un tratamiento eficaz. La socialización entre la comunidad médica y científica de las patologías poco frecuentes contribuye a la difusión del conocimiento y con él al incremento subyacente de la calidad de la atención al individuo, la familia y la comunidad.

\section{Referencias Bibliograficas.}

Arenas, C. F. (2011). El embarazo ectópico se incrementa en el mundo. Revista Cubana de Obstetricia y Ginecología. http://scielo.sld.cu/scielo.php?script=sci_arttext\&pid=S0138600X2011000100010

Burgos J, Ja, A., Albisu M, Corcostegui, M. J., Prieto B, Ramón O, \& Matorras R. (2004). Embarazo ectópico bilateral simultaneo tras IAC Simultaneous bilateral ectopic pregnancy after IUI (Vol. 21).

Carrillo, C. C. (2003). Epidemiología del embarazo ectópico en un hospital ginecoobstétrico. Revista Cubana de Enfermería. http://scielo.sld.cu/scielo.php?pid=S0864$03192003000300003 \&$ script=sci_arttext\&tlng=pt

Cearra, I., Da Silva, A., De Luis, N., \& Domínguez, I. (2009). Pasado, presente y futuro del embarazo ectópico. Gaceta Médica de Bilbao, 106(2), 53-60. https://doi.org/10.1016/s03044858(09)74647-5

Cristina González, B., Patricia Isabel Salas, B., Alicia Hernández, G., \& Javier De Santiago, G. (2012). Ovarian ectopic pregnancy: 10 years experience in the La Paz University Hospital, Madrid, Spain. En Revista Chilena de Obstetricia y Ginecologia (Vol. 77, Número 1, pp. 50-54). Sociedad Chilena de Obstetricia y Ginecologia. https://doi.org/10.4067/s071775262012000100010

Guerrero-Martínez;, E., \& RivasLópez, R. (2014). Algunos aspectos demográficos asociados con el embarazo ectópico. Ginecología y Obstetricia de México.

Hidalgo, N. R. (1995). Embarazo ectópico. Revista Cubana de Obstetricia y Ginecología. http://scielo.sld.cu/scielo.php?pid=S0138-600X1995000100001\&script=sci_arttext\&tlng=pt

Lozano, E. C., \& García, B. B. (2003). medigraphic.com Embarazo ectópico cervical. Informe de un caso. En Rev Med IMSS (Vol. 41, Número 6).

Pérez, C. B. (2002). Electron J Biomed 2013;3:63-66.- Carta. Bernárdez y col. EMBARAZO ECTÓPICO INTERSTICIAL. Revista de Obstetricia y Ginecología de Venezuela. https://biomed.uninet.edu/2013/n3/bernardez.html

Pinto, F. M., Bello, A. R., Gallardo-Castro, M., Valladares, F., Almeida, T. A., Tena-Sempere, M., \& Candenas, L. (2015). Analysis of the expression of tachykinins and tachykinin receptors in the rat uterus during early pregnancy. Biology of Reproduction, 93(2). 
https://doi.org/10.1095/biolreprod.115.130617

Radan, A., Aleksandra Polowy, J., Heverhagen, A., Simillion, C., Baumann, M., Raio, L., Schleussner, E., Mueller, M., \& Surbek, D. (2020). Cervico-vaginal placental $\alpha$ macroglobulin-1 combined with cervical length for the prediction of preterm birth in women with threatened preterm labor. Acta Obstetricia et Gynecologica Scandinavica, 99(3), 357-363. https://doi.org/10.1111/aogs.13744

Varona;, R. C. A. L. C. B. R. G. P. J., \& Martha Mohamed Abdelaziz. (2007). Embarazo ectópico cervical: Presentación de un caso. Revista Cubana de Obstetricia y Ginecología. http://scielo.sld.cu/scielo.php?pid=s0138-600x2007000100002\&script=sci_arttext\&tlng=en.

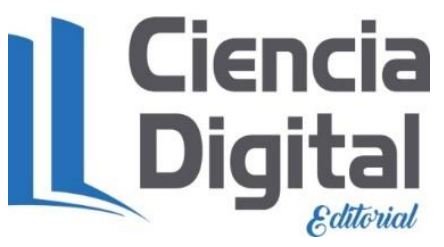


PARA CITAR EL ARTÍCULO INDEXADO.

Jordán Bolaños, A. I., Mantilla P, C. A., \& Rodríguez, C. E. (2020). Embarazo ectópico cervical: Estudio de un caso clínico. Anatomía Digital, 3(3), 82-88. https://doi.org/10.33262/anatomiadigital.v3i3.1391

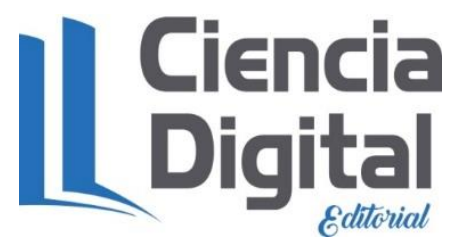

El artículo que se publica es de exclusiva responsabilidad de los autores y no necesariamente reflejan el pensamiento de la Revista Anatomía Digital.

El artículo queda en propiedad de la revista y, por tanto, su publicación parcial y/o total en otro medio tiene que ser autorizado por el director de la Revista Anatomía Digital.
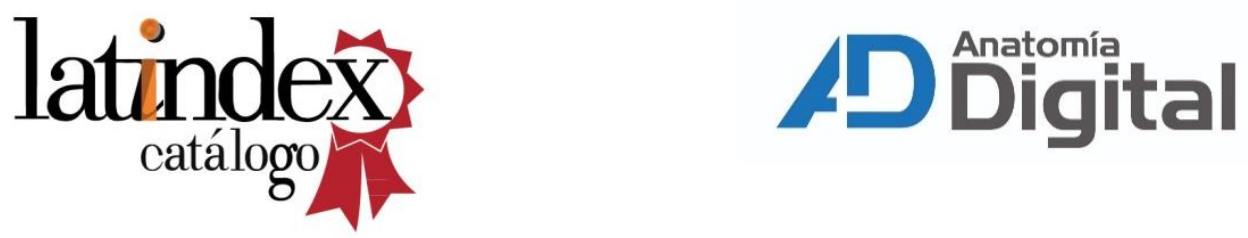